\title{
The Use of Kalman Filter Techniques for Ship Track Estimation
}

\author{
MANSOUR H. ASSAF ${ }^{1}$, VOICU GROZA ${ }^{2}$, and EMIL M. PETRIU ${ }^{2}$ \\ ${ }^{1}$ School of Engineering \& Physics, The University of the South Pacific, Suva, FIJI ISLANDS \\ assaf_m@usp.ac.fj \\ ${ }^{2}$ School of Information Technology and Engineering, Faculty of Engineering, \\ University of Ottawa, Ottawa, ON K1N 6N5, CANADA
}

\begin{abstract}
Unscented Kalman Filter (UKF) is a technique used in non-linear applications and dynamic systems identification (e.g. tracking marine vessels and ships) that require state and parameter estimation. This paper studies Kalman Filter (KF) based techniques for tracking ships using Global Positioning System (GPS) data. The present work proposes to exploit information from GPS sensors in order to track a ship in real-time. The absence and presence problem of a ship is handled by a applying KF theory to analyze GPS coordinates and compare current marine vessel routes to previously recorded ones.

To study tracking performance, the system was implemented in $\mathrm{C}++$ and simulation results demonstrate the feasibility and high accuracy of the proposed tracking method.
\end{abstract}

Keywords - Uncented Kalman Filter, tracking, sensors, Global Positioning System

Received: October 24, 2019. Revised: January 7, 2020. Accepted: January 31, 2020. Published: February 14, 2020.

\section{Introduction}

This paper proposes a tracking method for marine vessels using the UKF, and presents performance analysis.

Tracking commercial, passenger, and fishing marine vessels from satellites is done by transmitting their locations (GPS coordinates) and identities via a universal Automatic Identification System (AIS); as shown in figure 1 , the safety navigation system was originally developed as a collision avoidance tool to enable marine vessels to see each other more clearly in all weather conditions and improve the shared captured information about surrounding environments.

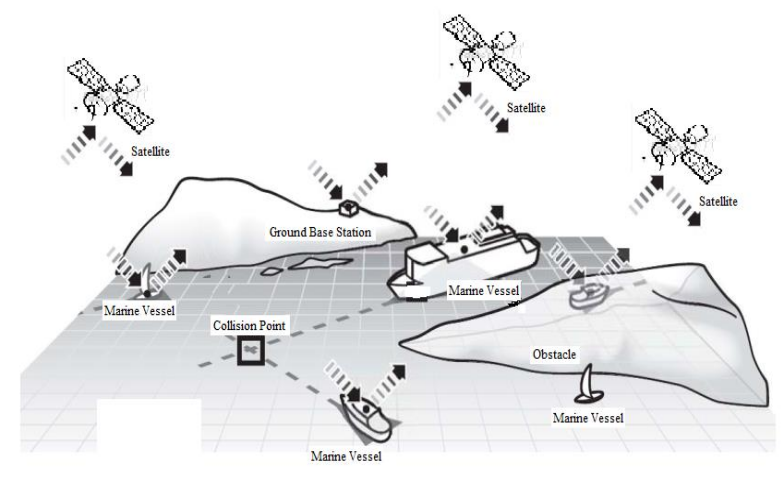

Fig. 1 AIS collision avoidance
The AIS continuously transmits a marine vessel's identity, position, and speed along with the course to all other AIS equipped vessels within range. The vessels' information is also transmitted to port authorities, via a shore station, to be able to manage maritime traffic and reduce marine navigation accidents.

It is estimated that over 130000 marine vessels are continuously broadcasting their locations over a 24 hour period. Since radar signals are usually refreshed every 5 seconds, and with such a large number of marine vessels, there are terabytes of data to be fed into computing systems for processing and analysis, which might become a challenging task to perform.

One other AIS challenging issue is that the transmitted radio signal (VHF band) is noisy. It is a tricky task to remove noise from information signal; one needs to know how much noise to remove in order to see the patterns of real ship data without removing so many ambiguous signals that may contain real data.

Near ports and where areas are crowded with vessels, communication bandwidth becomes 
congested with competing signals that interfere with one another. In addition, satellites and ground base receiving stations can handle limited amount of information at one time. Therefore any individual marine vessel may drop on and off the map because of this congestion. To improve coverage, more satellites can be deployed to receiver AIS signals, a scenario that would be very costly.

Besides, AIS signals may drop out causing long gaps in transmission. One of the main reasons is when a vessel captain turns the AIS signal off to avoid detection.

Occasionally, marine vessels' tracks appear in unusual locations i.e. over the mountains or in the deserts. The main two reasons for such cases are either the AIS transponder has had operation faults, or it has been tampered with deliberately.

Marine vessel operators have to input identity codes, including the vessel's name, type of boat and a Maritime Mobile Service Identity (MMSI) number, to their AIS by hand, which may lead to errors if it is left out, incomplete or deliberately entering false MMSI numbers to attempt at evading authorities.

Applying prediction and estimation techniques is needed to correct the position error and determine the vessel's true location.

Combined efforts of expert engineers and computer scientists with great skills and knowledge about marine vessel behavior, satellite signals and computer technology are put together to be able to identify which key pieces of information a computer needs in order to understand false signals, spoofed tracks and mistaken identity.

Kalman filter (KF) techniques have been successfully used to predict and estimate errors in position of linear systems [1]. The problem of marine vessel position estimation is nonlinear in nature; therefore modified KF techniques needed to be used for prediction of coordinates, namely the Uncented Kalman Filter (UKF), which has shown high performance especially in state-estimation of nonlinear applications, as been demonstrated in literature.

In this paper, we extend the use of the UKF to address a more general nonlinear estimation problem i.e. marine vessels tracking. In the next paragraph, we present key findings from a literature review on the application of tracking marine vessels.

\section{Background}

Trajectory tracking is a vital important issue for maneuvering and surveillance of marine vessels. A nonlinear vectorial back-stepping nonlinear modern control theory based method for tracking commercial ships was proposed in [4]. Tacking is accomplished by applying Lyapunov stability analysis corresponding to the state vectors dynamics, kinematics and actuator dynamics of the ships. A multivariate sliding mode control law method based on nonlinear horizontal motion dynamics of marine vessels with three degree of freedom, was proposed in [5]. As per this method, the positions and yaw angles of the ship are tracked simultaneously. In [6], an adaptive control with dynamic control allocation technique is proposed for the positioning of marine vessels. The positioning control method is developed to handle parametric uncertainties, unknown disturbances and input non-linearity. A method for detecting the absence or presence of a ship based on the fusion of raw data obtained from radar and an automatic identification system, and using a binary hypothesis testing problem, was proposed in [7]. An approach for determining the optimum track of ships was proposed in [8]. The authors propose a unique approach that takes into account both voluntary and involuntary speed reductions of the ship. The optimum route is obtained by using the inverse velocity as a weight function for the path and applying the Dijkstra's algorithm in an iterative manner.

Due to the non-linearity nature of the ship dynamic and kinematic problems, many proposed control design techniques are not competent enough for 
obtaining the optimal routes of marine vessels [12]. The application of the UKF based control techniques can be attractive and promising in this context. The UKF application areas may be divided into state-estimation and machine learning [2], which are briefly reviewed in the next paragraph.

\section{Overview}

Kalman Filter is a tool that predicts values using a bunch of mathematical equations under the assumptions that input data is in the form of Gaussian distribution and then followed by the application of linear equations to that Gaussian distribution.

In real world, many models (i.e. marine vessels) involve solving non linear equations such trigonometric functions (i.e. sine and cosine) due to fact that the system may be predicting in one direction while our sensor is taking reading in some other direction. Therefore the Extended Kalman Filter (EKF) takes helps of Taylor Series and Jacobian Matrix to linearly approximate a non linear function around the mean of the Gaussian and then predict the values [11].

As presented in literature, the EKF takes one point that is the mean of the Gaussian to approximate a new linear function from non linear function, approximation around the mean point. To achieve a better approximation, we can consider not only one point but a bunch of points including the mean and we approximate around those multiple points; the Unscented Kalman Filter (UKF) method. As the number of points in source Gaussian increases, computational power and resources increase, so a there is a compromise between the number of points taken and performance. The optimal solution is represented by a good approximation with a reasonable number of points with acceptable use of resources. The Weighted Sigma Points concept is applied by considering individual points on source Gaussian. These points have weights and they are mapped on target Gaussian after passing points through some non linear function, and then after the new mean and variance of transformed Gaussian are calculated. The sigma weighted points are the representatives of the whole Gaussian distribution. When a Gaussian is passed through a non linear function, it does not remain Gaussian anymore but it is an approximation of the Gaussian.

The steps are summarized as follows:

1. Compute Set of Sigma Points;

2. Assign Weights to each sigma point;

3. Transform the points through non linear function;

4. Compute Gaussian from weighted and transformed points;

5. Compute Mean and Variance of the new Gaussian.

The error in prediction is calculated by finding the cross-correlation between sigma points in state space and sigma points in the measurement space, as follows:

$$
T=\sum_{i=0}^{2 n} \omega^{[i]}\left(\chi^{[i]}-\mu^{\prime}\right)\left(z^{[i]}-\hat{z}\right)^{T}
$$

$S=\sum_{i=0}^{2 n} \omega^{[i]}\left(Z^{[i]}-\hat{\mathrm{z}}\right)\left(Z^{[i]}-\hat{\mathrm{z}}\right)^{T}+Q$

$K=T \cdot S^{-1}$

Where:

$\mathrm{T}$ is the cross-correlation matrix between state space and predicted space;

$\mathrm{Q}$ is the noise;

$\mathrm{n}$ is the dimensionality;

$\omega$ represents the weights of sigma points;

$\chi$ represents the sigma points matrix;

$\mathcal{Z}$ is the predicted measurement

$\mathrm{S}$ is the predicted covariance matrix; and

$\mathrm{K}$ is the Kalman filter gain.

To predict the final state after calculating the Kalman gain:

$\mu=\mu^{r}+K(z-\hat{z})$ 
$\Sigma=(I-K . T) \Sigma^{\prime}$

Where:

$\mu$ represents the mean;

$\Sigma$ is the covariance;

$\mu^{\prime}$ is the predicted mean;

$\Sigma^{\prime}$ is the predicted covariance;

$z$ represents the actual measurement mean coming from the sensor; and

$\hat{\mathrm{Z}}$ is the mean is measurement space.

In the following paragraph, we discuss the proposed marine vessel tracking procedure.

\section{Methodology}

The proposed system receive GPS data from satellites in real-time. The system processes GPS info, generates and interprets results.

Assuming that the earth is a spherical a circumference of 24,902 miles, GPS (sensor) position coordinates in degree measurements (1 degree $=60$ nautical miles) are located along a sphere that is divided into 180 degrees segments east and west of the prime meridian. At the equator, one nautical mile is equivalent to 30.86 meters. A one millimeter error is equal to 80 meters error on the real earth, using a 1:80000 scale. Marine vessel speed depends on the water current speed; it is measured in nautical miles per hour, and is represented by a vector quantity with magnitude and phase [9].

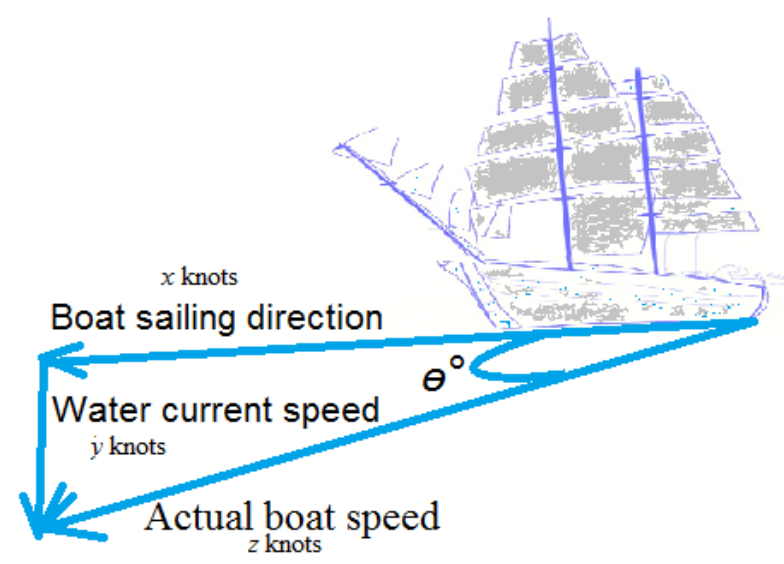

Fig. 2 Boat speed
As shown in figure 2, a ship sailing at speed of $\mathrm{x}$ knots heading southwest, and given a water current speed of $y$ knots to the south, the actual speed of the boat is of a $\mathrm{z}$ knots magnitude and a $\Theta^{\circ}$ angle.

The Unscented Kalman Filter (UKF) based GPS coordinates tracking procedure includes six main steps (figure 3):

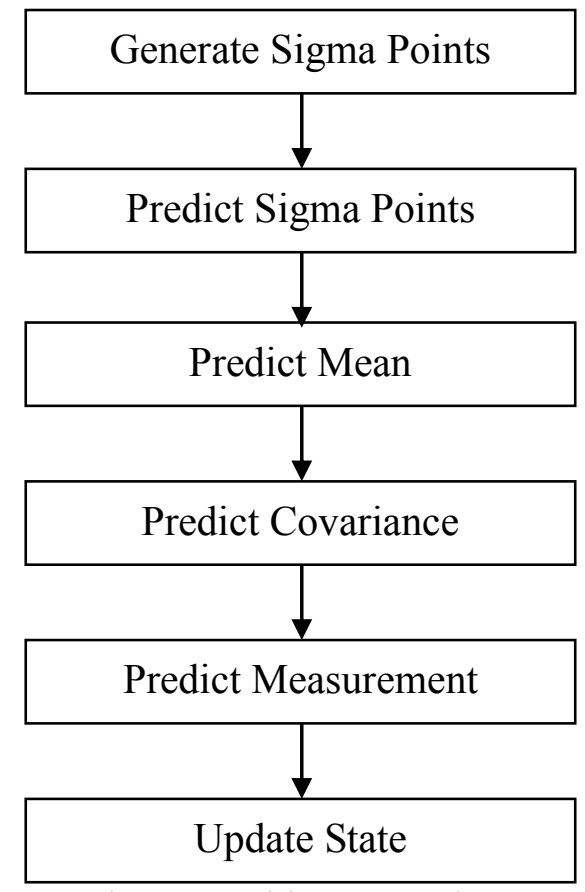

Fig. 3 Tracking procedure

The tracking algorithm is presented below as pseudocode:

1. given GPS sensor data measurement vector $z$ and timestamp $t$

2. if $1^{\text {st }}$ set of data is true initialize state vector $\mathrm{x}\left(p_{x}, p_{y}, \nu, \Psi, \Psi^{\prime}\right)$ and state covariance matrix $P$

else

compute the time difference $d t$ between previous $t_{0}$ and new $t_{1}$ time measurements

3. given current $x, P, d t$ get predicted_x, predicted_P, and predicted_sigma_x

4. given predicted_sigma_x 
get predicted_sigma_z, predicted_z, and

measured_covariance_S

5. given $z, \quad$ measured_covariance_S, predicted_P, predicted_x, predicted_z, predicted_sigma_x, and predicted_sigma_z get updated_P and updated_x

In the next section, we present the experimental results.

\section{Experimental Results}

To validate our proposed tracking algorithm, we have collected measured marine GPS data from various categories of marine vessels including passenger and commercial cargo ships, and fishing boats at different worldwide port locations and in different weather conditions. Next, we present two case studies in the Norwegian and Fijian coastal areas.

Case study 1 (Fiji islands): We collected and processed GPS data from marine vessel SUNSHINE 801, a Fijian fishing boat of 191 gross tonnage and $36.6 \mathrm{~m} \times 6 \mathrm{~m}$ overall length $\mathrm{x}$ breadth extreme, built in 2006 [12]. Figure 4 shown below displays a simple nautical map of the Fijian coastal area.

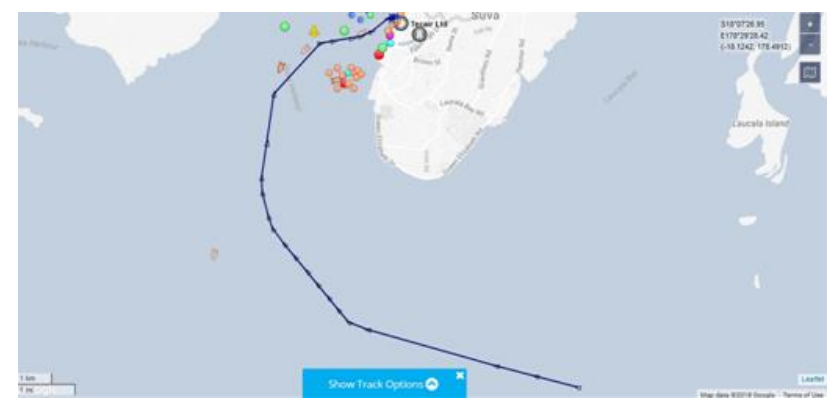

Fig. 4 Nautical map of Fiji [10]

Table 1 presents samples of input data including GPS coordinates, timestamp, speed, and course.

\begin{tabular}{|lllll|}
\hline \multicolumn{6}{|c|}{ Table 1 Sunshine $801[\mathrm{FJ}]$ - Input data [12] } \\
\hline \multicolumn{7}{|c|}{ Longitude $\left(^{\circ}\right)$ Latitude $\left({ }^{\circ}\right)$ Timestamp Speed $(\mathrm{Kn})$ Course $\left(^{\circ}\right)$} \\
\hline 177.4826 & -18.5299 & 1508937182 & 7.0 & 273 \\
177.4789 & -18.5354 & 1508938170 & 7.1 & 269 \\
177.4759 & -18.5411 & 1508938668 & 7.1 & 271 \\
177.4683 & -18.5470 & 1508940720 & 7.4 & 276 \\
177.4578 & -18.5506 & 1508941280 & 7.6 & 271 \\
177.4440 & -18.5540 & 1508946245 & 7.6 & 275 \\
177.4250 & -18.5579 & 1508946727 & 7.6 & 271 \\
177.4123 & -18.5607 & 1508947418 & 7.5 & 272 \\
177.3985 & -18.5636 & 1508948706 & 7.6 & 275 \\
177.3787 & -18.5679 & 1508952221 & 7.4 & 272 \\
177.3632 & -18.5716 & 1508953793 & 7.6 & 275 \\
\hline
\end{tabular}

Table 2 shows the longitude/latitude coordinates converted into $\mathrm{X}-\mathrm{Y}$ Cartesian coordinates and the projection of the speed along the $\mathrm{X}$ and $\mathrm{Y}$ axis.

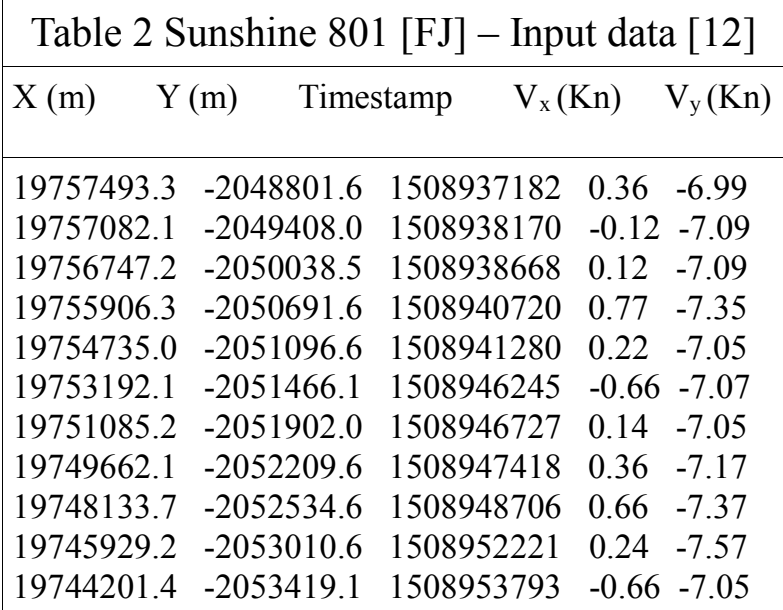

Figure 5 shown below displays the observed and estimated GPS coordinates after applying the Unscented Kalman Filter.

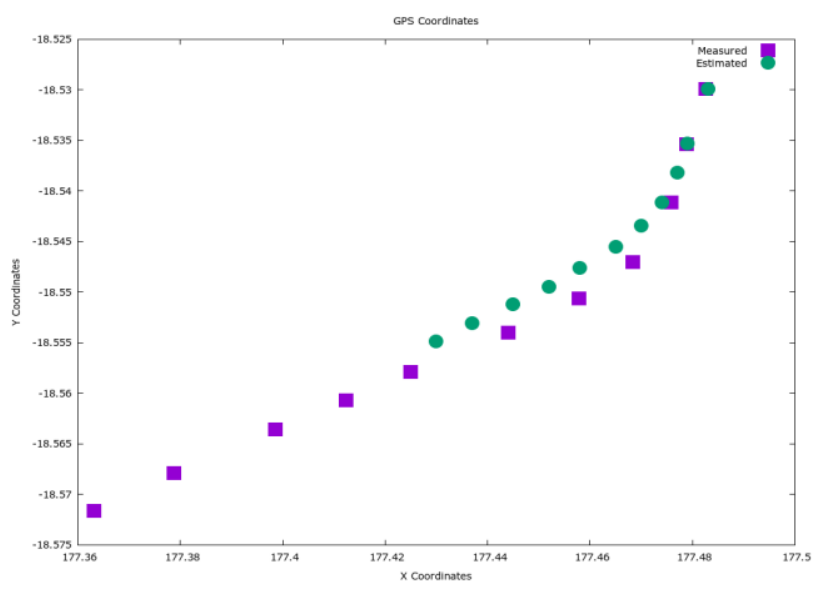

Fig. 5 Observed/Estimated GPS coordinates 
Solid squares in purple and solid circles in green represent the observed and estimated marine vessel positions, respectively. The rootmean-squared error of trajectory is used to measure accuracy; the error difference between observed and predicted positions and velocities is found to be $\left(\mathrm{X}, \mathrm{Y}, \mathrm{V}_{\mathrm{x}}, \mathrm{V}_{\mathrm{y}}\right)=(170.052$, $291.280,0.036,1.566)$.

Case study 2 (Norway): We have collected and processed GPS data from marine vessel YTTERQY, a Norwegian passenger ship of 1201 gross tonnage and $49.9 \mathrm{mx} 13.2 \mathrm{~m}$ overall length $\mathrm{x}$ breadth extreme, built in 2015 [12]. Figure 6 shown below displays a simple nautical map of the Norwegian Coastal area.

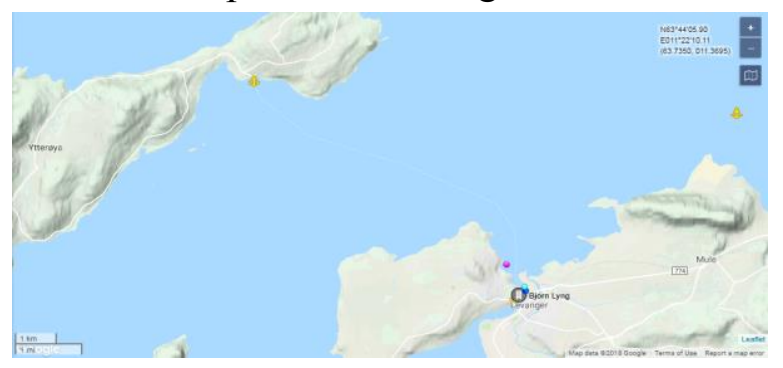

Fig. 6 Nautical map of Norway [10]

The observed and estimated tracks are shown in figure 7 below.

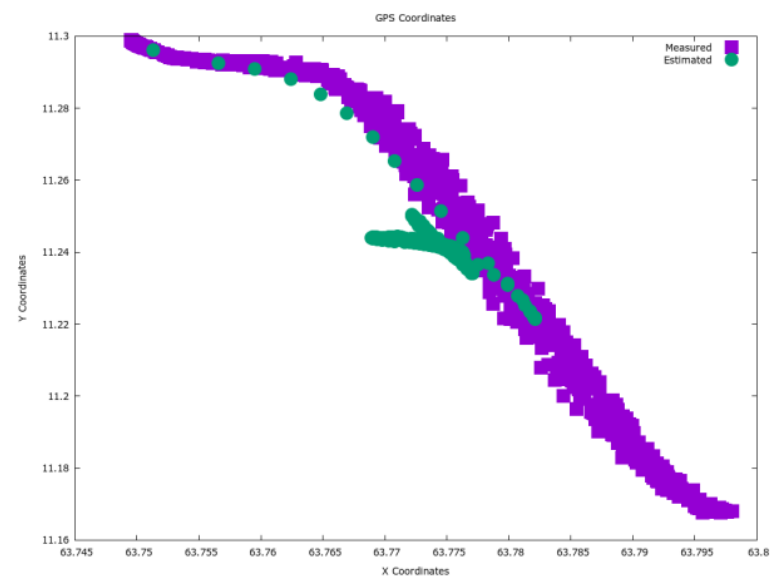

Fig. 7 YTTERQY marine vessel tracks

Solid squares in purple and solid circles in green represent the observed and estimated marine vessel positions, respectively. The error difference between observed and predicted positions and velocities is $\left(\mathrm{X}, \mathrm{Y}, \mathrm{V}_{\mathrm{x}}, \mathrm{V}_{\mathrm{y}}\right)=$ (55.716, 218.799, 0.025, 1.873).
Experimental results show that marine vessels estimated tracks follow the same pattern as the observed ones with acceptable error difference and better compared to the estimated errors reported in [12].

\section{Conclusion}

This paper discusses the design, implementation and validation of marine vessel tracking algorithm based on the Unscented Kalman Filter. Data representing various kinds of ships at different locations was used to validate the proposed algorithm and to show its effectiveness. Obtained results show that the proposed algorithm is promising and effective in tracking normal cruising speed. We plan to extend this work further by applying other techniques such as Particle Filter and Nonlinear Predictive Control to better optimize performance.

\section{References:}

[1] S. Haykin. Adaptive Filter Theory. Prentice-Hall, Inc, 3 edition, 1996.

[2] E.A. Wan, and R. Van Der Merwe, "The unscented Kalman filter for nonlinear estimation," IEEE Adaptive Systems for Signal Processing, Communications, and Control Symposium, Lake Louise, Alberta, Canada, 4 October 2000.

[3] A. Czapiewska, and J. Sadowski, "Algorithms for Ship Movement Prediction for Location Data Compression," International Journal on Marine Navigation and Safety of Sea Transportation, Vol. 9, No. 1, March 2015, pp. 75-81.

[4] T.I. Fossen, and S.P. Berge, "Nonlinear vectorial backstepping design for global exponential tracking of marine vessels in the presence of actuator dynamics," Proceedings of the 36th IEEE Conference on Decision and Control, San Diego, CA, USA, 12 December 1997.

[5] J. Cheng, J. Yi, and D. Zhao, "Design of a sliding mode controller for trajectory tracking problem of marine vessels," IET Control Theory \& Applications, Vol. 1, No. 1, January 2007, pp. 233 - 237.

[6] M. Chen, S. Sam Ge, B. Voon Ee How, and Y. Sang Choo, "Robust Adaptive Position Mooring Control for Marine Vessels," IEEE Trans. on Control Systems Technology, Vol. 21, No. 2, March 2013, pp. 395 - 409.

[7] F. M. Vieira, F. Vincent, J.-Y. Tourneret, D. Bonacci, M. Spigai, M. Ansart, and J. Richard, "Ship detection using SAR and AIS raw data for maritime 
surveillance," The 24th European Signal Processing Conference (EUSIPCO), Budapest, Hungary, 29 August - 2 September 2016.

[8] J. K. Panigrahi, C. P. Padhy, D. Sen, J. Swain, and O. Larsen, "Optimal ship tracking on a navigation route between two ports: a hydrodynamics approach," Journal of Marine Science and Technology, Springer, Vol. 17, No. 1, March 2012, pp. 59-67.

[9] D. Willemsen. (2017, July 2). Navigation course [Online]. Available: http://www.sailingissues.com/navcourse $0 . h t m l$

[10] Marine Traffic. (2017 Edition). Satellite AIS [Online]. Available: http://www.marinetraffic.com

[11] A. Germani, C. Manes, P. Palumbo, "Polynomial extended Kalman filter," IEEE Transactions on Automatic Control, Vol. 50, No. 12, December 2005, pp. 2059 - 2064.

[12] M. H. Assaf, E. M. Petiu, and V. Groza, "Ship Track Estimation Using GPS Data and Kalman Filter", Proc. I²MTC 2018 - IEEE Int. Instrum. Meas. Technol. Conf., Houston, Texas, USA, 14-17 May 2018. 